\title{
Local Heat Transfer Coefficient Measurements, Using a Transient Imaging Method With an Inverse Scheme
}

\author{
A. EL ABBADI, D. BOUGEARD, B.BAUDOIN \\ Ecole des Mines de Douai, Département Energétique Industrielle, \\ 941, rue Charles Bourseul, B.P 838, .59508 DOUAl, Cedex, France, \\ e-mail: el-abbadi@ensm-douai.fr
}

\begin{abstract}
Local heat transfer coefficient (LHTC) on heat exchangers fin surfaces is usually measured by means of transient or steady methods. Usually, in transient method, it's determined using the assumption of pure convective flux ( no lateral conduction in fin).

However this assumption is not totally validates since a lateral conduction flux still exists inside the fins. We present in this paper several improvements we brought to this transient method and particularly the use of inverse 2-D scheme to correct LHTC obtained without taking into account the lateral heat conduction.

The inverse scheme is implemented and then validated using a numerical test case with a specific LHTC variation. Then the method is used in a real experiment to determine LHTC on a single finned tube.
\end{abstract}

\section{Introduction}

The estimation of convective heat transfer distribution is a main task in designing compact heat exchangers. For instance the knowledge of local heat transfer coefficient is of a particular importance to design new heat transfer surface for highperformance heat exchanger.

Usually two main methods have been used to determine local heat transfer on surfaces:

\section{- The steady state}

- Transient method

The first methods was used by Eibeck and Eaton [1], Pauley and Eaton [2] and consist of a constant heat flux surfaces with thermocouple array in a steady state condition. This steady method was also used by Herchang Ay et al [3] to measure local heat transfer coefficient of plate finned-tube heat exchanger by infrared thermography. Steady state heat transfer with a constant flux density is conceptually simple and easy to analyse but the provision of a constant flux surface poses experimental difficulties. The use of electrically heated foils is possible, but uniform flux density can be difficult to achieve in practice.

On the other hand, transient methods using Infrared Thermography or Thermochromic Liquid Crystals are reported by Ireland and Jones [4], Dong et al [5] and Tourreuil and Bougeard [6]. They offers advantages of flexibility because it being comparatively easy to alter the geometry of the surface being studied.

In the study of Tourreuil et al [6], Fiebig et al[7], the transient technique was used in the case of a thin wall with a very low thermal conductivity in order to neglect the conduction flux along the wall. They investigate numerically this assumption of negligible heat conduction along the wall. Their computation show that significant heat conduction for a thin wall of low thermal conductivity occurs only at the positions where the distribution of heat transfer coefficients has local extrema with large second derivatives. There the local experimental error due to heat conduction can reach $10 \%$.

However, to study and develop enhanced surfaces of heat exchangers, a best knowledge of local heat transfer coefficient is required to determine the optimal values of these enhanced surfaces parameters.

We present in this paper a transient method and visualization technique using infrared thermography. And an inverse 2-D scheme to take into account the lateral conduction along the fin surface in order to obtain local heat transfer coefficient on compact heat exchangers surfaces. 


\section{Transient Infrared Thermography Method}

\section{1 Temperature measurement of the fin surface}

Our investigation technique (more detailed in our precedent paper El Abbadi et al[8]) requires an accurate knowledge of the spatial distribution of the fin surface temperature. This measurement is made with an infrared camera (AGEMA 900 Long Wave) equipped with an HgCdTe sensor cooled by a Stirling motor. The infrared camera is placed at its minimal distance in order to give maximal spatial resolution (see figure 1).The electromagnetic energy radiated in the infrared spectral band by an element of the fin is converted into an electronic signal from the camera sensor. Then this signal is amplified and digitally recorded with a 12-bit dynamic range. In order to convert this signal (expressed by numeric level (NL)) into temperature, an in-situ calibration is made using a thermal sensor. The calibration procedure use a special high thermal conductivity plate coated with the same black painting as the fin, and placed at the same location. In order to enhance the thermal resolution, the calibration curve uses the whole dynamic range (12 bit) of temperature variation measurement of the model during the experiment.

More over the spatial resolution is enhanced using a specific image restoration technique [8].

Thanks to these various treatments, our experimental technique allows accurate measurements of the fin temperature with an overall uncertainty of about $\pm 0.3^{\circ} \mathrm{C}$.
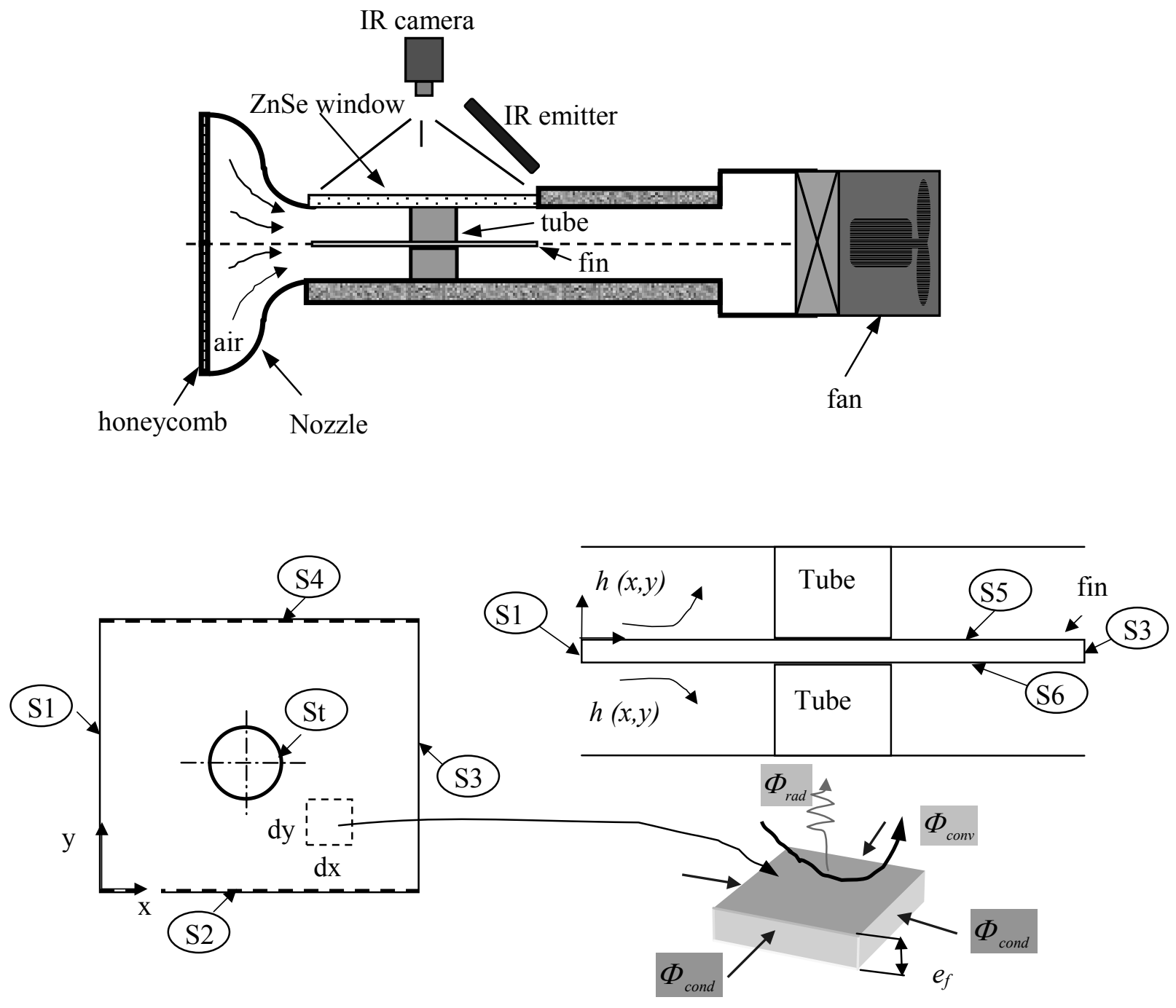

Fig.1. experimental test bench and test model 


\section{2 Local heat transfer calculation with the assumption of negligible heat conduction}

The heat transfer measurement is performed using a transient technique based on the work of Ireland and Jones [4]. The knowledge of the time temperature variation on the fin surface allows to calculate the heat flux in each small element of the fin and hence the heat transfer coefficient.

The heat fluxes balance in an element gives:

$$
m_{f} \frac{\partial H_{f}}{\partial t}=\Phi_{c o n v}+\Phi_{c o n d}+\Phi_{r a d}
$$

in order to simplify the problem several assumption could be made :

- constant temperature across the plate thickness (i.e., negligible temperature gradient in the $\mathrm{z}$ direction) this assumption is validated because the thickness and material of the fin are chosen in order to gives a Biot number value under 0.1 .

- $\quad$ negligible radiative fluxes.

- constant heat transfer coefficient during the experiment.

The precedent equation becomes:

$$
\rho_{f} C p_{f} e_{f} d x d y \frac{\partial T_{f}}{\partial t}=2 h(x, y) d x d y\left(T_{i n}-T_{f}\right)+\lambda_{f} \rho_{f} d x d y\left(\frac{\partial T^{2}}{\partial x^{2}}+\frac{\partial T^{2}}{\partial y^{2}}\right)
$$

Usually researchers neglect the term of conduction and integrate equation 3 between 0 and $t_{f}$ to get simplified expression of LHTC:

$$
h_{\text {ln }}(x, y)=\frac{\rho_{f} C p_{f} e_{f}}{2 \Delta t} \times \ln \left(\frac{T_{i n}-T_{f}(x, y, 0)}{T_{i n}-T_{f}\left(x, y, t_{f}\right)}\right)
$$

Since the plate is cooled from both sides, Eq. (3) gives the heat transfer coefficient locally averaged for the upper and lower sides of the plate.

\section{3 Correction of local heat transfer measurement}

In real problem, $h(x, y)$ is not known and must be determined from transient temperature measurements on the fin surface. This kind of problem is similar to an inverse conduction problem.

More precisely in our study the unknown LHTC is determined using an iterative process (written in Matlab.6 code) as described bellow :

- Firstly we determine the LHTC using the assumption of negligible heat conduction (eq 3).

- Secondly the direct 2-D heat conduction scheme is used to determine the time variation temperature field. The general governing equations is of this $2-D$ problem is (eq 2), where $h$ is the coefficient calculated by mean of the expression (eq 3). The numerical calculation was performed using the second-order central differencing scheme for spatial axis and implicit method for temporal step.

- Thirdly, for the time, $t_{f}$, corresponding to the experimental acquisition of the temperature filed at the end of the experiment, the calculated temperature, Tfc, is compared to the experimental one. The comparison criterion is

$$
C(x, y)=T_{f_{\exp }}(x, y)-T_{f_{c}}(x, y)
$$

If the value of this criterion is as great as 0.1 , the new local heat transfer is then calculated by the relation:

$$
h^{\prime}(x, y)=h(x, y)+\alpha \times \operatorname{sign}(C(x, y)) \times h(x, y)
$$

Where $\alpha$ is a correction coefficient lower than $10^{-1}$ and "sign" is a Matlab6 function which gives de sign of the array C. The correction loop continues until the calculated transient temperature information of each pixel on the fin surface matches the experimental data within the convergence criterion. 


\section{http://dx.doi.org/10.21611/qirt.2004.042}

\section{4 Validation of the inverse scheme}

First of all, this inverse scheme was validated using a numerical test case before its use to calculate the heat transfer coefficient on fin surface from experimental data.

The numerical test model chosen is formed from a flat plate without tube. Its thermal conductivity was varied from 0.1 (PVC) to $100 \mathrm{~W} / \mathrm{m} . \mathrm{K}$ (steel).

The flow boundary and initial conditions are chosen as follow:

- $\quad$ Given heat transfer coefficient on the faces S5 and S6 (see Fig 2,3)

$$
\begin{array}{ll}
h(x, y)=10+a b s(60 \cos (x)) & \text { if } \quad(x, y) \in\left[0: \frac{\pi}{2}, 0: \frac{\pi}{2}\right]-\left[\frac{\pi}{5}: \frac{3 \pi}{10}, \frac{\pi}{5}: \frac{0.9 \pi}{2}\right] \\
h(x, y)=c s t e=80 & \text { if } \quad(x, y) \in\left[\frac{\pi}{5}: \frac{3 \pi}{10}, \frac{\pi}{5}: \frac{0.9 \pi}{2}\right]
\end{array}
$$

- $\quad$ Adiabatic surfaces S1, S2, S3 and S4.

- $\quad$ Uniform initial temperature on fin surfaces S5 and S6.

The direct scheme is used to calculate the cooling of the fin due to the LHTC given above.

Figures (2,3 and 4$)$ show the real LHTC, the estimated one $\left(h_{l n}\right)$ calculated using equation 3 and the calculated one using the inverse scheme. We can clearly see that $h_{l n}$ is very different from the real imposed coefficient $h$.

As shown in figure 5 real heat transfer coefficient was successfully recovered, by mean of this inverse scheme method.

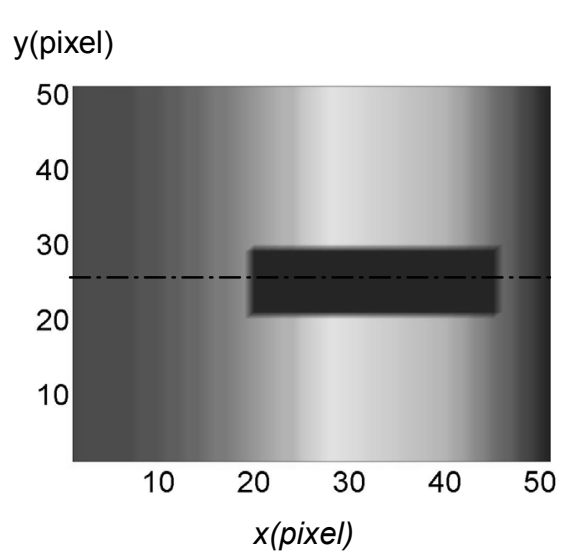

Fig.2. real $h(x, y)$

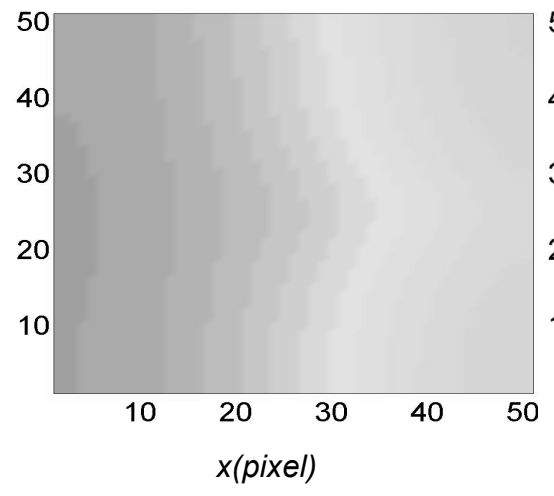

Fig.3. $h_{\ln }(x, y)$ with the assumption of negligible conduction flux

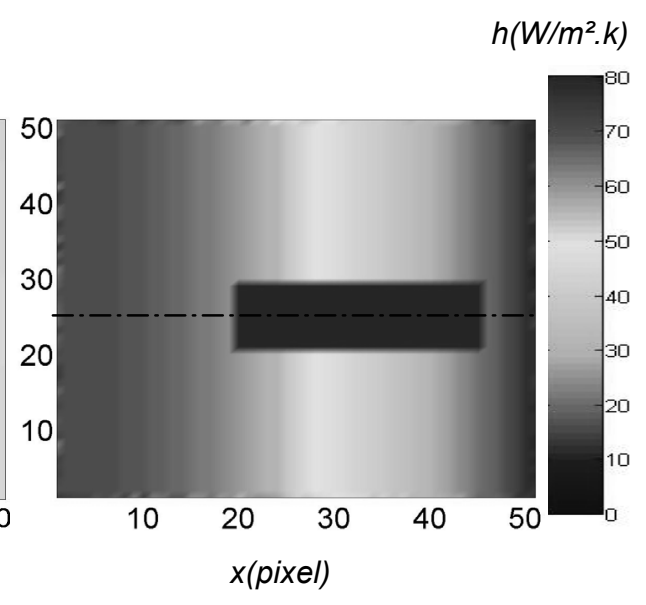

Fig.4. $h(x, y)$ corrected with the inverse method

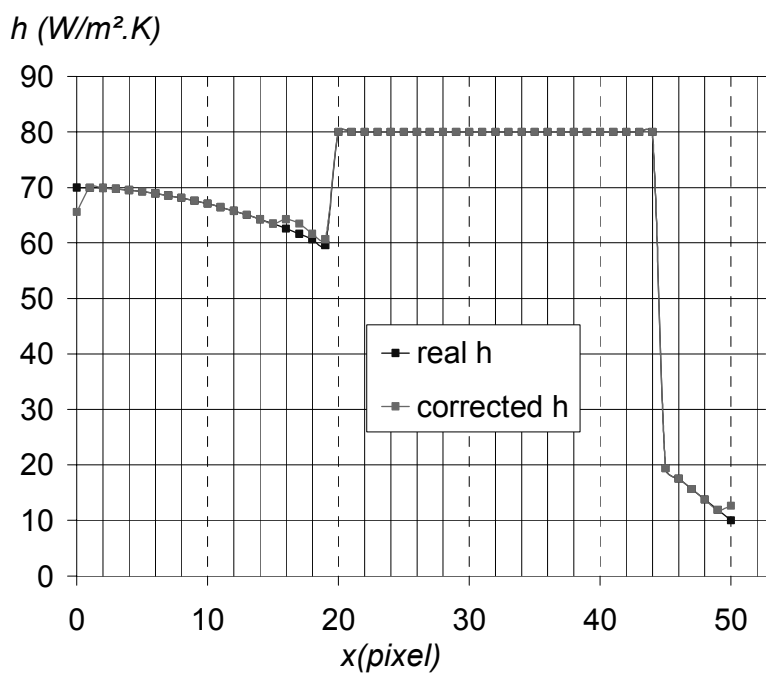

Fig.5. real and corrected LHTC along the fin center line 


\section{5 Experimental validation}

Once this method was numerically validated, it was applied on a real case. Figure 6 represent a map of heat transfer coefficient obtained experimentally on a model of a finned tube. The fin used, which is made of a PVC material, has an thermal conductivity of $0.1 \mathrm{~W} / \mathrm{m}$.K. More detail about this study is published in [8].

On the Figure 7, we plot the real and corrected local heat transfer coefficient along the tube center line. As we can note, globally there isn't a big difference between the two coefficients because of the low thermal conductivity of the fin. But in the area where LHTC has extrema, the deviation is more visible.

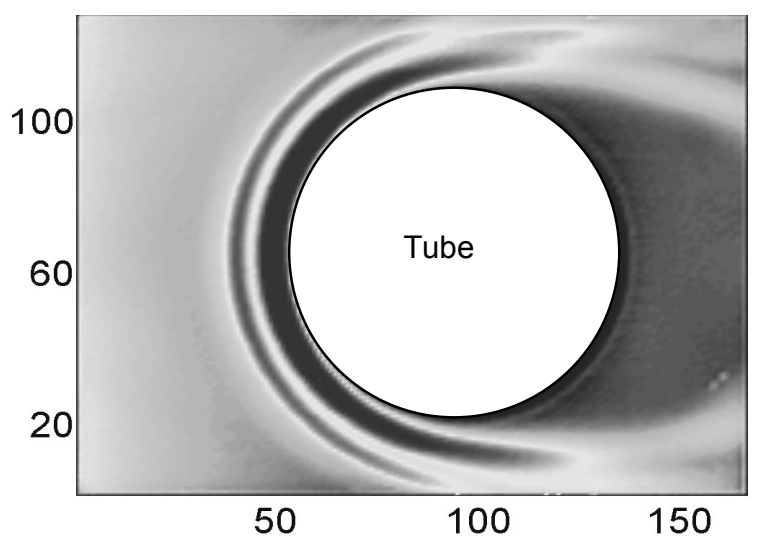

Fig.6. map of $h$ on a finned tube

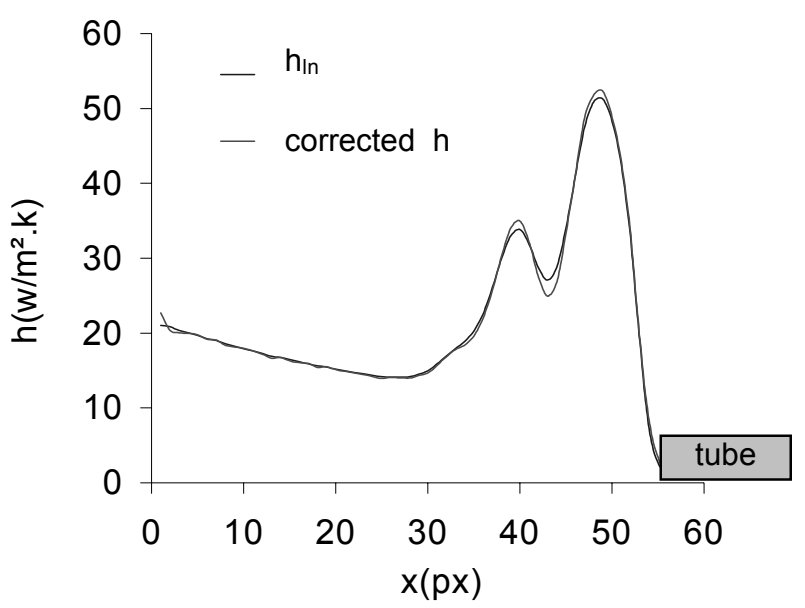

Fig.7. $h$ along the tube center line

This technique, gives a good results in the cases of low thermal conductivities fin $(0-10 \mathrm{~W} / \mathrm{m} . \mathrm{K})$. But in the case of fins of high thermal conductivities, a filtration technique of the temperature must be used because of the noise of experimental temperature.

Our inverse technique could be improved using a filtering technique like the one used by S.Rainieri and G.Pagliarini [9] to estimate local heat transfer coefficient in the presence of noise.

\section{Conclusion}

A transient Infrared Thermography method was developed to measure the local heat transfer coefficient on finned tube heat exchangers.

The local heat transfer coefficient calculated without taking into account the conduction along the fin surface was corrected by mean of an inverse 2-D conduction scheme.

This inverse scheme was numerically validated before its test on a finned tube of low thermal conductivity.

On these low thermal conductivity fins, results show that the error on global heat transfer is not important if we neglect the conduction but it's not the case for local heat transfer coefficient.

This method can also be used for a fin with a relatively high thermal conductivity $0-10 \mathrm{w} / \mathrm{m}^{2} . \mathrm{K}$ but for a very high thermal conductivity material, a filtration technique is needed.

\section{Nomenclature}

Bi Biot number $\left(=h e_{f} / 2 \lambda_{f}\right)$

$\beta \quad$ Coefficient of thermal expansion per unit volume, $\left[\mathrm{K}^{-1}\right]$

$C p_{f} \quad$ Fin specific heat, $\mathrm{J} / \mathrm{kg} \mathrm{K}$

$\Delta t \quad$ Cooling Time, $\mathrm{s}$

$e_{f} \quad$ Fin thickness, $\mathrm{m}$

$g \quad$ Gravity, $\mathrm{m} / \mathrm{s}^{2}$

$h \quad$ Heat transfer coefficient, $\mathrm{W} / \mathrm{m}^{2} \mathrm{~K}$ 


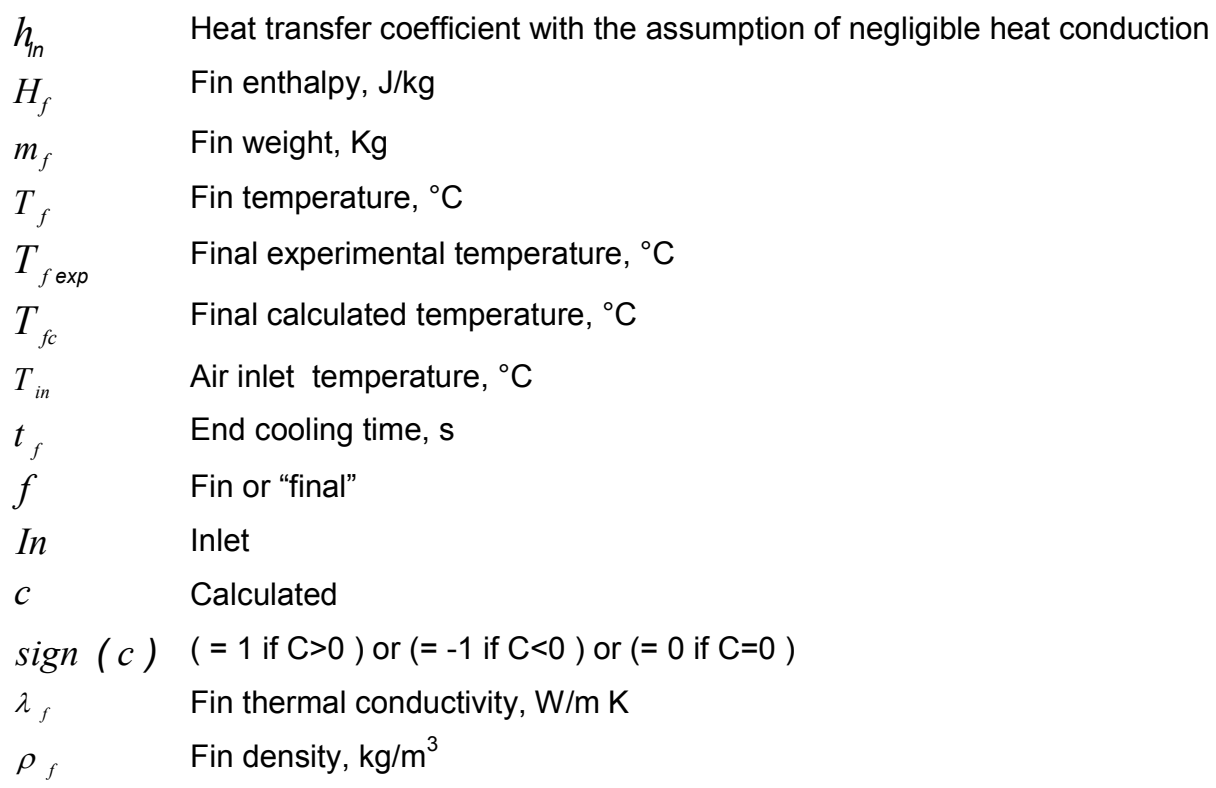

\section{REFERENCES}

[1] P.A. Eibeck, J.K. Eaton, - "Heat transfer effects of longitudinal vortex embedded in a turbulent boundary layer", ASME J. Of Heat transfer 109, 16:23, 1987

[2] W.R Pauley, J.K. Eaton, - "The effect of longitudinal embedded vortex arrays on turbulent boundary layer heat transfer", ASME J. Of Heat transfer 116, 871:879, 1996

[3] Herchang Ay, J.Y.Jang, J.N.Yeh, - "Local heat transfer measurements of plate finned-tube heat exchangers by infrared thermography", IJMHT 45, 4069:4078, 2002

[4] P.T. Ireland, T.V. Jones - "Detailed measurements of heat transfer on and around a pedestal in fully developed channel flow", 8 th International heat transfer conference, Vol. 3, San Francisco, 975 - 980, 1986

[5] Y. Dong, S. Tiggelbeck, M. Fiebig, N. K. Mitra, - "Influence of delta-winglet vortex generators on heat transfer and drag of a fin-tube", Eurotherm Seminar ${ }^{\circ}$ 9, Bochum, Heat Transfer in Single phase flows, July 10-11, 1989

[6] J. Tourreuil, D. Bougeard, B. Baudoin, -“Influence of conjugate heat transfer in compact heat exchangers with vortex generators", $3^{\text {rd }}$ International Conference on Compact Heat Exchangers and Enhancement Technology for the Process Industries, p. 57-64, Davos, 2001, Begell House Inc., ISBN 1-56700-164-5

[7] M. Fiebig, A. Valencia, N. K. Mitra, - "Local heat transfer and flow losses in fin-and-tube heat exchangers with vortex generators : a comparison of round and flat tubes", Experimental thermal and Fluide sciences, 8:35-45, 1994

[8] A. El abbadi, D.Bougeard, B.Baudoin - "Experimental Study of Heat Transfer Distribution on a Single Finned Tube", 4 th European Thermal Sciences Conference, Birmingham , UK, 2004

[9] Sara Rainieri. Fabio Bozzoli, Giorgio paliarini - "Wiener filtering technique applied to themographic data reduction intended for the estimation of plate fins performance", Experimental thermal and Fluide sciences 28 , 179:183, 2004 